International Journal of Psychological Research and Reviews
(ISSN:2639-6041)

\title{
Loneliness in Adults During a COVID-19 Lockdown
}

\section{Sonia S. Rehman*, Shantay Mines*, Samantha Poling*, Tiffany Field*'**, Debra Bendell* \& Connie. S. Veazey*}

${ }^{*}$ Fielding Graduate University; ** University of Miami/Miller School of Medicine

\begin{abstract}
Loneliness has been frequently reported in the COVID-19 literature but rarely in the context of potential comorbidities including depression, fatigue, and sleep problems. The present study assessed the relationships between self-reported loneliness, and the potential comorbidities based on an archival database called the COVID-19 Lockdown Activities Survey ( $N=260$ participants). Loneliness was endorsed by $67 \%$ of the participants. Greater loneliness was related to greater depression, fatigue, and sleep problems. A regression analysis revealed that depression contributed to $47.1 \%$ of the variance in loneliness. These results highlight the multidimensionality of loneliness. The generalizability of these results is limited by the homogeneity of the sample (predominantly non-Hispanic white females). Nonetheless, they highlight the importance of designing interventional protocols that address loneliness and the associated depression, fatigue, and sleep problems rather than loneliness alone.
\end{abstract}

Keywords: COVID-19 Lockdown, Loneliness, Depression, Fatigue, Sleep Problems
${ }^{*}$ Correspondence to Author:

Sonia S. Rehman

Fielding Graduate University

How to cite this article:

Sonia S. Rehman, Shantay Mines, Samantha Poling, Tiffany Field , Debra Bendell \& Connie. S. Veazey.Loneliness in Adults During a COVID-19 Lockdown. International Journal of Psychological Research and Reviews, 2021, 4:55

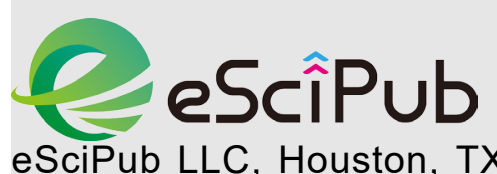
eSciPub LLC, Houston, TX USA. Website: https://escipub.com/ 
Mental health research on COVID-19 lockdowns suggests several prevalent problems, including anxiety and depression (Bartoszek et al., 2020; Luo et al., 2020), touch deprivation (Field, Poling, et al., 2020), sleep disorders (Partinen, 2021), higher perceived social isolation, substance use, and decreased life satisfaction (Clair et al., 2021), lower levels of spiritual wellbeing (Coppola et al., 2021), posttraumatic stress symptoms (Liu et al., 2020), fatigue (Bartoszek et al., 2020) and loneliness (Bartoszek et al., 2020; Field, et al., 2020). The mental health challenges associated with COVID-19 are significant, and specific interventions have not appeared in the literature. A recent news story suggested that a "mental health version of Dr. Anthony Fauci is missing."(Lewinsky, 2020, p. 1).

\section{Loneliness}

Although the telecommunication revolution of the 21st century has connected individuals globally (Sapolsky et al., 2020), loneliness is also on the rise (Sweet, 2020). According to a National Survey by Cigna, $61 \%$ of individuals in the US reported feeling lonely (Novotney, 2019). In another recent study, data from individuals between the ages of 16 and 99 who lived across 237 geographic regions in the world suggested a significant prevalence of loneliness (Barreto et al., 2021). The results further suggested that men experience loneliness more than women, that loneliness decreases when adolescents reach adulthood, and that participants from individualistic cultures report it more frequently. It is noteworthy that loneliness has been perceived as social isolation. However, individuals can live alone but not feel lonely or live an active social life and still feel alone. Consequently, loneliness is defined as a perceived experience of separation from others (Rokach, 2019).

Further, loneliness affects individuals' happiness, cognitive functioning, and physical health (Yeh, 2017). Centers for Disease Control and Prevention (CDC) describes loneliness and social isolation as a serious public health risk (Centers for Disease Control and Prevention
[CDC], 2021). The prevalence of loneliness worldwide highlights the importance of researching loneliness and its comorbid mental health problems.

\section{Depression}

A recent study by CDC showed that fluctuations in COVID-19 cases resemble the reports of anxiety and depression from week to week (Stephenson, 2021). A meta-analysis of global community-based studies during the first seventeen weeks of 2020 documented the prevalence of depression to be about seven times higher (about 25\%) than pre-COVID estimates (about 3.44\%) (Bueno-Notivol et al., 2021). A review from India, Pakistan, and Bangladesh estimated the pooled prevalence of depression to be $34.1 \%$ in 28 studies (Hossain et al., 2021). Similarly, in a longitudinal study from Australia, the mean scores for depression and anxiety showed at least mild symptoms (Batterham et al., 2021). These studies suggest that the COVID-19 pandemic has been associated with depression worldwide.

\section{Fatigue}

A recent meta-analysis of 15 studies $(\mathrm{N}=47,910)$ showed that most patients diagnosed with COVID-19 continue to report physical and cognitive symptoms during their post-viralinfection follow-up visit. Fatigue is the most reported post-COVID symptom at an estimated prevalence of $58 \%$ (Stengel et al., 2021). Fatigue has also been reportedly the most debilitating symptom that persists after the acute phase of COVID-19 (Rudroff et al., 2020). The World Health Organization-Europe reported that although most public support COVID-19 response strategies of maintaining social distance and wearing masks, pandemic fatigue is also emerging rapidly (World Health Organization [WHO], 2020). In addition, fatigue and burnout are well documented in healthcare workers during COVID-19 surges, especially in high-volume intensive care units (Sasangohar et al., 2020).

\section{Sleep Problems}


A systematic review and meta-analysis involving 168 studies from 39 countries estimated that about $31 \%$ of healthcare professionals, $18 \%$ of the general population, and $57 \%$ of COVID-19 patients struggle with sleep-related issues (Alimoradi et al., 2021). Sleep problems were associated with depression in this analysis. Another study showed a prevalence of sleep problems in about $40 \%$ of the general public and health care providers (Jahrami et al., 2021).

\section{Purpose of the Present Data Analyses}

The purpose of the present data analyses was to assess the relationships between selfreported loneliness, depression, fatigue, and sleep problems based on an archival database of a COVID-19 lockdown survey. In addition, analyses were conducted to determine the relative variance in loneliness that was explained by depression, fatigue, and sleep problems. Feelings of loneliness were expected to be associated with greater depression, fatigue, and sleep problems.

Methods

\section{Participants}

A $G^{*}$ power analysis indicated that a sample size of 224 was required for an alpha of .05 and $80 \%$ power. The participants included individuals $(\mathrm{N}=260)$ who ranged from $18-82$ ( $M=47$ years). Gender was distributed $79 \%$ female, $18 \%$ male and 3\% other (non-specified). Ethnicity was distributed $68 \%$ Non-Hispanic White, 21\% Hispanic, 3\% Black and 8\% other (non-specified). Professions were distributed 35\% office worker, 30\% academic, 15\% managerial, $12 \%$ medical and $8 \%$ labor. The average income was $\$ 72,572$, 28\% were unemployed and $69 \%$ worked at home. Twenty-three percent lived alone.

\section{Procedure}

A flyer was posted on Facebook giving a brief description of the study, including some sample items and the age criterion being greater than 18 years. The Facebook flyer included a link to Survey Monkey, an online survey development cloud-based software. The survey was conducted between April 1, 2020, and April 30, 2020 , and the data were directly transported to SPSS for data analyses.

\section{Measures}

The survey included several demographic items, including age, gender, ethnicity, profession, income, type of employment, working at home, and living alone. Depression, fatigue, and sleep problems were assessed using those subscales of the Patient-Reported Outcomes Measurement Information System (PROMIS) (Cella et al., 2010). PROMIS Depression subscale includes four items, such as "I feel depressed," rated on a 5-point Likert-type scale ranging from $1=$ never to $5=$ always with an alpha of .91(Teresi et al., 2016). Fatigue was measured on the three-item PROMIS Fatigue subscale that includes statements such as "I felt fatigued," with a reported alpha of .88 (Ameringer et al., 2016). The PROMIS sleep subscale consist of five items on the same Likert scale with an alpha of .86 (Yu et al., 2012). These items include statements like "My sleep quality was bad". Loneliness was assessed using a single item, "I feel lonely," on a 4-point Likert scale.

\section{Results}

A multiple regression analysis was conducted to determine the variance in loneliness that was explained by the PROMIS depression, sleep and fatigue subscales. As can be seen in Table 1, the three predictor variables explained $48.4 \%$ of the variance in loneliness and the model was significant $(R 2=.484, F(3,249)=77.81, p<.001)$. Depression was a significant predictor of Ioneliness $(t=9.77, p<.001, \beta=0.17)$ but sleep $(t=0.67, p=.504, \beta=0.01)$ and fatigue $(t=1.80$, $p=.074, \beta=0.04$ ) were not.

To find the best model, a stepwise multiple regression was conducted. In the first model, depression as a predictor explained $47.1 \%$ of total variance, $F(1,251)=223.87 ; p<.001$. In the second model, introducing the fatigue variable explained an additional $1.2 \%$ of the variance in loneliness, and the change in $\mathrm{R}^{2}$ was 
significant, $F(1,250)=5.55, p=.019$. The result of the third model suggests that if fatigue increases for one unit, loneliness will increase for 0.05 . The nature of the predictive value of depression was as expected; if depression increases for one unit, loneliness will increase for 0.17 .

A one-way ANOVA was conducted to examine whether participants' depression, fatigue, and sleep differed depending on the level of feeling lonely ( $1=$ Not at all to $3=\mathrm{A}$ lot). The results indicate differences in mean depression $\mathrm{F}(3$, $253)=77.34, p<.001$, fatigue $F(3,254)=32.67$, $p<.001$ and sleep $F(3,249)=18.80, p<.001$ as a function of the loneliness ratings.

\section{Table 1 Result of Multiple Regression Analysis}

\begin{tabular}{|c|c|c|c|c|c|c|}
\hline \multirow[b]{2}{*}{ Predictor } & \multicolumn{2}{|c|}{$\begin{array}{l}\text { Unstandardized } \\
\text { Coefficients }\end{array}$} & \multicolumn{2}{|c|}{$\begin{array}{l}\text { Standardized } \\
\text { Coefficients }\end{array}$} & \multirow[b]{2}{*}{$R^{2}$} & \multirow[b]{2}{*}{$F$} \\
\hline & $b$ & $S E$ & $\beta$ & $t$ & & \\
\hline Model 1 & & & & & .484 & $77.81^{\star \star}$ \\
\hline Constant & 0.34 & .17 & & $2.00^{*}$ & & \\
\hline Depression & 0.17 & .02 & .59 & $9.77^{\star \star}$ & & \\
\hline Fatigue & 0.04 & .02 & .12 & 1.80 & & \\
\hline Sleep Problems & 0.01 & .01 & .04 & 0.67 & & \\
\hline tMode 2 & & & & & .471 & $223.87^{\star *}$ \\
\hline Constant & 0.60 & .12 & & $4.88^{\star \star}$ & & \\
\hline Depression & 0.20 & .01 & .69 & $14.96^{* *}$ & & \\
\hline tMode 3 & & & & & .483 & $116.74^{* *}$ \\
\hline Constant & 0.40 & .15 & & & & \\
\hline Depression & 0.17 & .02 & .60 & $10.16^{\star \star}$ & & \\
\hline Fatigue & 0.05 & .02 & .14 & $2.36^{\star \star}$ & & \\
\hline
\end{tabular}

Table 2 Mean scale scores for significant ANOVAs for loneliness, depression, fatigue, and sleep problems (standard deviations in parentheses).

\begin{tabular}{lllllllll}
\hline & \multicolumn{7}{c}{ Loneliness Level } \\
\cline { 2 - 8 } Variables & \multicolumn{1}{c}{$(0)$ Not at all } & 1 & & 2 & & (3) a lot & \\
\cline { 2 - 8 } & $M(S D)$ & Pvalue & $M(S D)$ & Pvalue & $M(S D)$ & Pvalue $M(S D)$ & Pvalue \\
\hline Depression & $5.61(2.21)$ & $<.001$ & $7.48(2.76)$ & $<.001$ & $9.79(3.07)$ & $<.001$ & $12.92(3.62)$ & $<.001$ \\
Fatigue & $6.11(2.47)$ & $<.001$ & $7.99(2.70)$ & $<.001$ & $9.09(2.50)$ & .04 & $10.55(3.02)$ & $<.001$ \\
Sleep Problems & $11.67(4.32)$ & $<.001$ & $13.43(3.88)$ & $<.001$ & $14.76(4.19)$ & .05 & $17.00(4.16)<.001$ \\
\hline
\end{tabular}

Post Hoc tests conducted using the Tukey HSD test suggest that participants who felt lonely "a lot" $(\mathrm{M}=12.92, \mathrm{SD}=3.62)$ had higher depression subscale scores than participants who gave ratings of $2(M=9.79, S D=3.07),(p<.001)$ or 1 $(M=7.48, S D=2.76),(p<.001)$ or those who did not feel lonely $(M=5.61, S D=2.21),(p=.001)$. In addition, participants who felt lonely "a lot" $(M=10.55, S D=3.02)$ resulted in significantly higher fatigue subscale scores than participants who gave ratings of $2(M=9.09, S D=2.50)$, $(p=.04)$ or $1(M=7.99, S D=2.70),(p<.001)$, or 
those who did not feel lonely ( $M=6.11, S D=2.47$ ), $(p<.001)$. Finally, participants who felt lonely "a lot" $\quad(M=17.00, \quad S D=4.16) \quad$ experienced significantly higher sleep problems subscale scores than participants who gave ratings of 2 $(M=14.76, S D=4.19),(p=.05)$ or $1(M=13.43$, $\mathrm{SD}=3.88$ ), or those who did not feel lonely $(\mathrm{M}=11.67, \mathrm{SD}=4.32),(\mathrm{p}<.001)$.

\section{Discussion}

These results show that depression explained a significant amount of variance in feelings of loneliness while fatigue and sleep problems did not. These findings correspond with the results of the previously mentioned study in which high levels of depressive symptoms and loneliness were found in a nationally representative probability sample of American adults (Rosenberg et al., 2021). Additionally, in the current study, greater loneliness was related to greater depression, fatigue, and sleep problems. These data support the previously reported relationship between fatigue, depression, sleep problems, and loneliness (Alimoradi et al., 2021; Barreto et al., 2021; Bueno-Notivol et al., 2021; Mayne et al., 2021; \& Stengel et al., 2021).

These results highlight the multidimensionality of loneliness and are a part of an important trend that attempts to explore the emotional and environmental contributors of loneliness. However, it is noteworthy that participants of our study were predominantly non-Hispanic white females. Thus, the results of the nonrepresentative sample would not be generalizable to the larger population or other countries. Future studies may utilize more heterogeneous populations or investigate more environmental variables as covariates.

Currently, the Delta variant is more infectious and is easily transmissible, even in some vaccinated individuals (Mahase, 2021). This transmissibility is causing uncertainty about the length of COVID-19 modified lifestyles. Although there is limited research on interventions to protect the psychological health of individuals who continue to quarantine and social distance, mindfulness-based interventions have previously demonstrated a significant improvement in wellbeing ((Field et al., 2021; Zhang et al., 2021). Therefore, intervention protocols should be designed to address not only loneliness but the associated depression, fatigue, and sleep problems as well. Interventions that address this combination of variables may be more effective than focusing on loneliness alone during pandemic lockdowns like those of COVID-19.

\section{References}

[1]. Alimoradi, Z., Broström, A., Tsang, H. W., Griffiths, M. D., Haghayegh, S., Ohayon, M. M., Lin, C.-Y., \& Pakpour, A. H. (2021). Sleep problems during covid-19 pandemic and its' association to psychological distress: A systematic review and meta-analysis. EClinicalMedicine, $\quad 36, \quad 100916$. https://doi.org/10.1016/j.eclinm.2021.100916

[2]. Ameringer, S., Elswick, R. K., Menzies, V., Robins, J., Starkweather, A., Walter, J., Gentry, A., \& Jallo, N. (2016). Psychometric evaluation of the patient-reported outcomes measurement information system fatigue-short form across diverse populations. Nursing Research, 65(4), 279-289.

https://doi.org/10.1097/nnr.0000000000000162

[3]. Barreto, M., Victor, C., Hammond, C., Eccles, A., Richins, M. T., \& Qualter, P. (2021). Loneliness around the world: Age, gender, and cultural differences in loneliness. Personality and Individual Differences, 169, 110066. https://doi.org/10.1016/j.paid.2020.110066

[4]. Bartoszek, A., Walkowiak, D., Bartoszek, A., \& Kardas, G. (2020). Mental well-being (depression, loneliness, insomnia, daily life fatigue) during covid-19 related homeconfinement-a study from poland. International Journal of Environmental Research and Public Health, 17(20), 7417. https://doi.org/10.3390/ijerph17207417

[5]. Batterham, P. J., Calear, A. L., McCallum, S. M., Morse, A. R., Banfield, M., Farrer, L. M., Gulliver, A., Cherbuin, N., Rodney Harris, R. M., Shou, Y., \& Dawel, A. (2021). Trajectories of depression and anxiety symptoms during the covid-19 pandemic in a representative australian adult cohort. Medical Journal of Australia, 214(10), 462-468. https://doi.org/10.5694/mja2.51043

[6]. Bueno-Notivol, J., Gracia-García, P., Olaya, B., Lasheras, I., López-Antón, R., \& Santabárbara, J. (2021). Prevalence of depression during the 
covid-19 outbreak: A meta-analysis of community-based studies. International Journal of Clinical and Health Psychology, 21(1), 100196.

https://doi.org/10.1016/j.ijchp.2020.07.007

[7]. Cella, D., Riley, W., Stone, A., Rothrock, N., Reeve, B., Yount, S., Amtmann, D., Bode, R., Buysse, D., Choi, S., Cook, K., DeVellis, R., DeWalt, D., Fries, J. F., Gershon, R., Hahn, E. A., Lai, J.-S., Pilkonis, P., Revicki, D.,... Hays, R. (2010). The patient-reported outcomes measurement information system (PROMIS) developed and tested its first wave of adult selfreported health outcome item banks: 20052008. Journal of Clinical Epidemiology, 63(11), 1179-1194.

https://doi.org/10.1016/j.jclinepi.2010.04.011

[8]. Centers for Disease Control and Prevention. (2021, April 30). Loneliness and social isolation linked to serious health conditions. https://www.cdc.gov/aging/publications/features /lonely-older-adults.html

[9]. Clair, R., Gordon, M., Kroon, M., \& Reilly, C. (2021). The effects of social isolation on wellbeing and life satisfaction during pandemic. Humanities and Social Sciences Communications, 8(1). https://doi.org/10.1057/s41599-021-00710-3

[10]. Coppola, I., Rania, N., Parisi, R., \& Lagomarsino, F. (2021). Spiritual well-being and mental health during the covid-19 pandemic in italy. Frontiers in Psychiatry, 12. https://doi.org/10.3389/fpsyt.2021.626944

[11]. Field, T., Mines, S., Poling, S., Diago, M., Bendell, D., \& Veazey, C. (2020, October 30). Young, alone, and young alone during a covid19 lockdown. Journal of Mental Health and Clinical Psychology. https://www.mentalhealthjournal.org/articles/yo ung-alone-and-young-alone-during-a-covid-19lockdown.html

[12]. Field, T., Poling, S., Mines, S., Bendell, D., \& Veazey, C. (2020). Touch deprivation and exercise during the covid-19 lockdown april 2020. Medical Research Archives, 8(8). https://doi.org/10.18103/mra.v8i8.2204

[13]. Hossain, M., Rahman, M., Trisha, N., Tasnim, S., Nuzhath, T., Hasan, N., Clark, H., Das, A., McKyer, E. J., Ahmed, H., \& Ma, P. (2021). Prevalence of anxiety and depression in south asia during covid-19: A systematic review and meta-analysis. Heliyon, 7(4), e06677. https://doi.org/10.1016/j.heliyon.2021.e06677

[14]. Jahrami, H., BaHammam, A. S., Bragazzi, N., Saif, Z., Faris, M., \& Vitiello, M. V. (2021). Sleep problems during the covid-19 pandemic by population: A systematic review and metaanalysis. Journal of Clinical Sleep Medicine, $17(2)$, 299-313. https://doi.org/10.5664/jcsm.8930

[15]. Lewinsky, M. (2020, October 1). The forgotten fword in the pandemic. Vanity Fair. https://www.vanityfair.com/news/2020/09/monic a-lewinsky-on-covid-mental-health-czar

[16]. Liu, N., Zhang, F., Wei, C., Jia, Y., Shang, Z., Sun, L., Wu, L., Sun, Z., Zhou, Y., Wang, Y., \& Liu, W. (2020). Prevalence and predictors of ptss during covid-19 outbreak in china hardesthit areas: Gender differences matter. Psychiatry Research, 287, 112921. https://doi.org/10.1016/j.psychres.2020.112921

[17]. Luo, M., Guo, L., Yu, M., Jiang, W., \& Wang, H. (2020). The psychological and mental impact of coronavirus disease 2019 (covid-19) on medical staff and general public - a systematic review and meta-analysis. Psychiatry Research, 291, 113190.

https://doi.org/10.1016/j.psychres.2020.113190

[18]. Mahase, E. (2021). Delta variant: What is happening with transmission, hospital admissions, and restrictions? BMJ, n1513. https://doi.org/10.1136/bmj.n1513

[19]. Novotney, A. (2019, May). The risks of social isolation [APA Monitor]. https://www.apa.org. https://www.apa.org/monitor/2019/05/cecorner-isolation

[20]. Partinen, M. (2021). Sleep research in 2020: Covid-19-related sleep disorders. The Lancet Neurology, 20(1), 15-17. https://doi.org/10.1016/s1474-4422(20)30456-7

[21]. Rokach, A. (2019). Loneliness, illness, and death: Universal experiences we dread. In The psychological journey to and from loneliness (pp. 21-44). Elsevier. https://doi.org/10.1016/b978-0-12-815618$6.00002-3$

[22]. Rosenberg, M., Luetke, M., Hensel, D., Kianersi, S., Fu, T., \& Herbenick, D. (2021). Depression and loneliness during april 2020 covid-19 restrictions in the united states, and their associations with frequency of social and sexual connections. Social Psychiatry and Psychiatric Epidemiology. https://doi.org/10.1007/s00127020-02002-8

[23]. Rudroff, T., Fietsam, A. C., Deters, J. R., Bryant, A. D., \& Kamholz, J. (2020). Post-covid-19 fatigue: Potential contributing factors. Brain Sciences, $\quad 10(12), \quad 1012$. https://doi.org/10.3390/brainsci10121012

[24]. Sapolsky, H. M., Crane, R. J., Neuman, W. R., \& Moan, E. M. (Eds.). (2020). The telecommunications revolution: Past, present 
and future (routledge library editions: The economics and business of technology) (1st ed.). Routledge.

[25]. Sasangohar, F., Jones, S. L., Masud, F. N., Vahidy, F. S., \& Kash, B. A. (2020). Provider burnout and fatigue during the covid-19 pandemic: Lessons learned from a high-volume intensive care unit. Anesthesia \& Analgesia, 131(1), 106-111. https://doi.org/10.1213/ane.0000000000004866

[26]. Stengel, A., Malek, N., Zipfel, S., \& Goepel, S. (2021). Long haulers-what is the evidence for post-covid fatigue? Frontiers in Psychiatry, 12. https://doi.org/10.3389/fpsyt.2021.677934

[27]. Stephenson, J. (2021). CDC study finds worsening anxiety and depression, especially in young adults, during covid-19 pandemic. JAMA Health Forum, 2(4), e210724. https://doi.org/10.1001/jamahealthforum.2021.0 724

[28]. Sweet, J. (2020, December 7). The loneliness pandemic. Harvard Magazine. https://www.harvardmagazine.com/2021/01/fea ture-the-loneliness-pandemic

[29]. Teresi, J. A., Ocepek-Welikson, K., Kleinman, M., Ramirez, M., \& Kim, G. (2016). Psychometric properties and performance of the Patient Reported Outcomes Measurement Information System ${ }^{\circledR}$ (PROMIS ${ }^{\circledR}$ ) depression short forms in ethnically diverse groups. Psychological Test and Assessment Modeling, 58(1), 141-181. https://pubmed.ncbi.nlm.nih.gov/28553573/

[30]. World Health Organization. (2020). Pandemic fatigue: Reinvigorating the public to prevent COVID-19

[PDF]. https://apps.who.int/iris/bitstream/handle/10665 /335820/WHO-EURO-2020-1160-4090655390-eng.pdf

[31]. Yeh, C. S. (2017, January 13). The power and prevalence of loneliness - harvard health blog harvard health publishing. Harvard Health Blog. https://www.health.harvard.edu/blog/the-powerand-prevalence-of-loneliness-2017011310977

[32]. Yu, L., Buysse, D. J., Germain, A., Moul, D. E., Stover, A., Dodds, N. E., Johnston, K. L., \& Pilkonis, P. A. (2012). Development of short forms from the promis ${ }^{\mathrm{TM}}$ sleep disturbance and sleep-related impairment item banks. Behavioral Sleep Medicine, 10(1), 6-24. https://doi.org/10.1080/15402002.2012.636266

[33]. Zhang, D., Lee, E. P., Mak, E. W., Ho, C. Y., \& Wong, S. S. (2021). Mindfulness-based interventions: An overall review. British Medical Bulletin, 138(1), 41-57. https://doi.org/10.1093/bmb/ldab005 